\title{
Nutritional B vitamin deficiency alters the expression of key proteins associated with vascular smooth muscle cell proliferation and migration in the aorta of atherosclerotic apolipoprotein $\mathbf{E}$ null mice
}

\author{
Susan J. Duthie - John H. Beattie • Margaret-J. Gordon • Lynn P. Pirie • \\ Fergus Nicol • Martin D. Reid • Gary J. Duncan • Louise Cantlay • \\ Graham Horgan • Christopher J. McNeil \\ Received: 3 June 2014/ Accepted: 21 November 2014/Published online: 2 December 2014 \\ (C) Springer-Verlag Berlin Heidelberg 2014
}

\begin{abstract}
Low B vitamin status is linked with human vascular disease. We employed a proteomic and biochemical approach to determine whether nutritional folate deficiency and/or hyperhomocysteinemia altered metabolic processes linked with atherosclerosis in ApoE null mice. Animals were fed either a control fat (C; $4 \% \mathrm{w} / \mathrm{w}$ lard) or a high-fat [HF; $21 \% \mathrm{w} / \mathrm{w}$ lard and cholesterol $(0 / 15 \%$ w/w)] diet with different $\mathrm{B}$ vitamin compositions for 16 weeks. Aorta tissue was prepared and global protein expression, B vitamin, homocysteine and lipoprotein status measured. Changes in the expression of aorta proteins were detected in response to multiple $\mathrm{B}$ vitamin deficiency combined with a high-fat diet $(P<0.05)$ and were strongly linked with lipoprotein concentrations measured directly in the aorta adventitia $(P<0.001)$. Pathway analysis revealed treatment effects in the aorta-related primarily to cytoskeletal organisation, smooth muscle cell adhesion and invasiveness (e.g., fibrinogen, moesin, transgelin, vimentin). Combined B vitamin deficiency induced striking
\end{abstract}

Electronic supplementary material The online version of this article (doi:10.1007/s12263-014-0446-y) contains supplementary material, which is available to authorized users.

S. J. Duthie $(\square)$

School of Pharmacy and Life Sciences, Robert Gordon

University, Aberdeen, UK

e-mail: s.j.duthie@rgu.ac.uk

S. J. Duthie - J. H. Beattie - M.-J. Gordon .

L. P. Pirie · F. Nicol - M. D. Reid · G. J. Duncan - L. Cantlay ·

C. J. McNeil

Division of Lifelong Health, Rowett Institute of Nutrition

and Health, University of Aberdeen, Aberdeen, UK

G. Horgan

Biomathematics and Statistics Scotland (BioSS),

Aberdeen AB21 9SB, UK quantitative changes in the expression of aorta proteins in atherosclerotic ApoE null mice. Deregulated expression of these proteins is associated with human atherosclerosis. Cellular pathways altered by B vitamin status included cytoskeletal organisation, cell differentiation and migration, oxidative stress and chronic inflammation. These findings provide new insight into the molecular mechanisms through which B vitamin deficiency may accelerate atherosclerosis.

Keywords Aorta proteome - ApoE null mice . Atherosclerosis - B vitamins - Hyperhomocysteinemia

\section{Introduction}

Low dietary folate is strongly associated with an increased risk of human vascular diseases including atherosclerosis and stroke (Voutilainen et al. 2000, 2004; Durga et al. 2005). This has been attributed primarily to suboptimal intracellular folate, together with low levels of vitamins $\mathrm{B}_{12}$ and $\mathrm{B}_{6}$, inducing hyperhomocysteinemia (Splaver et al. 2004). However, there is evidence for folate alone influencing human vascular disease. High circulating folate is associated with reduced risk of primary coronary events and carotid intima-media thickness independently of total plasma homocysteine in several prospective human studies (Voutilainen et al. 2000, 2004; Durga et al. 2005). Moreover, supplemental folic acid positively influences vascular function in patients with, or at risk of, vascular disease (Mangoni et al. 2005; Tawakol et al. 2005; Till et al. 2005). These effects appear largely independent of homocysteine.

Determining whether low folate or hyperhomocysteinemia is causal for vascular disease in vivo is difficult due to the intimate metabolic relationship linking folate and 
homocysteine. Transgenic mouse strains provide a representative model of human atherosclerosis and allow complete investigation of the effect of diet on vascular disease progression. The ApoE knockout mouse spontaneously develops atherosclerotic plaques that are morphologically analogous to lesions found in human tissue (Zhang et al. 2002). We have developed distinct nutritional deficiencies in transgenic ApoE null mice so that they exhibit either mild or moderate hyperhomocysteinemia (McNeil et al. 2011). We have shown that $B$ vitamin deficiency increases atherosclerotic plaque formation in transgenic ApoE knockout mice and that this is strongly linked with accumulation of cholesterol and pro-atherogenic lipoproteins in the adventitial lipid surrounding the aorta (McNeil et al. 2011, 2012).

Proteomic technologies have been widely used to identify quantitative changes in protein expression as a consequence of several human vascular pathologies including atherosclerosis, aorta medial degeneration and myocardial revascularisation (Wu et al. 2007; Delbosc et al. 2008; Farina et al. 2010). Moreover, they have proven to be powerful tools in rodents studies for providing mechanistic insight into how vascular disease progresses and is influenced by environmental factors such as diet (Mayr et al. 2005; Almofti et al. 2006).

Here, we employed a combined biochemical and quantitative global proteomics approach to determine how prolonged nutritional folate deficiency and/or moderate hyperhomocysteinemia differentially modulates global protein expression directly in the aorta of ApoE null mice and to further identify whether these changes are associated with proteins and processes involved in the progression of human atherosclerosis.

\section{Materials and methods}

Animal study and diets

The design of this animal study and the composition of the experimental diets employed have been described in detail elsewhere (McNeil et al. 2011, 2012). All procedures were carried out in accordance with the requirements of the UK Animals (Scientific Procedures) Act 1986. ApoE null mice (males, 5 weeks old) were allocated (by weight) to one of six treatment groups ( $n=10$ animals per group). Diets were based on either a control [C; $4 \% \mathrm{w} / \mathrm{w}$ lard] or high-fat [HF; $21 \% \mathrm{w} / \mathrm{w}$ lard and cholesterol $(0.15 \% \mathrm{w} / \mathrm{w})]$ background with different B vitamin compositions applied to each (1) folic acid and B vitamin replete, $(2)$ folic acid depleted $(\mathrm{F}-)$, or (3) folic acid, $\mathrm{B}_{6}$ and $\mathrm{B}_{12}$ depleted ( $\left.\mathrm{F}-\mathrm{B}-\right)$. Mice were fed these diets for 16 weeks.
Blood and aorta collection

Mice were fasted up to $6 \mathrm{~h}$ and killed by exsanguination under terminal anaesthesia. Blood was collected from the vena cava for analysis of B vitamins, homocysteine and lipoproteins. Residual blood was flushed from the heart by injection of Dulbecco's modified Eagles medium (DMEM) into the left ventricle. The complete aorta was microdissected of tunica adventitia. The thoracic and abdominal aorta and the dissected adventitia were snap-frozen and stored at $-80{ }^{\circ} \mathrm{C}$ for proteomic and lipid analysis. The aortic arch was fixed in $10 \%$ formalin and atherosclerotic plaque volume quantified by staining plaques in situ with the neutral lipid-targeting lysochrome Oil Red O (ORO, Sigma, Poole, UK), which targets fatty acid deposits in the arterial plaques and solubilising and measuring spectrophotometrically the dye retained in the aorta. Solubilised ORO as a biomarker of atherosclerotic plaque volume was corrected for aorta tissue weight (Beattie et al. 2009; McNeil et al. 2011, 2012).

Preparation of aorta tissue for proteomic analyses

Weighed frozen aorta (approx. $10 \mathrm{mg}$ ) was added to extraction buffer containing $7 \mathrm{M}$ urea, $2 \mathrm{M}$ thiourea, $4 \%$ CHAPS and $2 \%$ BioRad Biolite Ampholyte pH 3-10 and homogenised using a Precellys 24 lysis and homogenisation cycler (Stretton Scientific, UK). The homogenate was centrifuged at $14,000 \mathrm{rpm}$ for $5 \mathrm{~min}$ at $4{ }^{\circ} \mathrm{C}$ and the supernatant frozen at $-80^{\circ} \mathrm{C}$. Protein was measured using the BioRad RC DC protein assay.

\section{D gel electrophoresis of aorta tissues}

One 2D gel was run per aorta $(100 \mu \mathrm{g})$ sample $(n=10$ mice per treatment group; 60 gels in total). Proteins were separated by isoelectric focusing in the first dimension [BioRad immobilised $\mathrm{pH}$ gradient (IPG) strips (pi range 3-10)] and SDS-PAGE in the second dimension on 8-16 acrylamide gels $(18 \times 18 \mathrm{~cm})$ as described previously (Duthie et al. 2008). Gels were stained with Coomassie blue and imaged on a BioRad GS710 flat bed imager followed by image analysis using PD Quest version 8.0.1. Spots were excised from the gels using a robotic BioRad spot cutter, trypsinised in a MassPrep Station (Waters, MicroMass, Manchester, UK) and analysed by LC MS/MS using an Ultimate nano LC capillary chromatography system (LC Packings, Camberly, Surrey, UK), combined with an Applied Biosystems 2000 Q-Trap (Warrington, UK). Peak list were generated using Analyst 1.4.2 software set at default. Peptide fragment mass spectra yielded a sequence of separated peptides that were pasted into the fingerprinting web resource programme Mascot dll version 1.6b9 (Matrix Science Ltd, Boston, MA) 
for protein identification. Protein identification was made using SWISS-PROT (version 2012.09) specifically for Mus musculus (mouse), with expectation values, cut-off scores and threshold values for individual proteins considered. Protein confidence parameters including Mowse scores, expected and actual masses, PI, number of unique peptides matched, percentage sequence coverage and gene and protein accession numbers, together with protein function, are shown for each identified peptide in the experimental results (Supplementary Table 1).

\section{B vitamin and lipoprotein analyses}

Blood and tissue folate and $\mathrm{B}_{12}$ were measured by radioassay and plasma total homocysteine by gas chromatography (McNeil et al. 2011, 2012). Lipid was extracted from adventitial tissue (approx. $200 \mathrm{mg}$ ) (Folch et al. 1957) and total cholesterol, LDL cholesterol, HDL cholesterol, TG and NEFA fractions measured in plasma and aorta adventitial lipid using a Konelab 20 Clinical Chemistry Analyser (Thermo Scientific, Passau, Germany). Fatty acids were trans-esterified to methyl esters (FAMEs) in methanolic HCL, separated by liquid-liquid solvent extraction and measured by gas-liquid chromatography (GLC; Burdge et al. 2000).

\section{Statistical analysis}

For proteomic analysis, 2D gel images were studied using PDQuest software (BioRad). After normalisation and matching, spot densities were exported for data analysis (multivariate) using Excel and "R" (R Foundation for Statistical Computing, Vienna, Austria; version 2.10). Log transformed spot densities from aorta tissue were analysed by two-way ANOVA for the effect of fat intake, vitamin composition and interaction between these factors $(P<0.05)$. These analyses provide quantitative protein expression data, as densitometry of the Coomassie-stained spots is carried out for each animal in each treatment group individually prior to statistical analysis.

Biochemical data were analysed by two-way ANOVA for the effect of fat intake, vitamin composition and interaction between these factors. Significant differences between all groups were detected using the least significant difference (LSD) post hoc method. Analysis of associations among aorta tissue protein expression and blood B vitamin status markers, blood and vascular lipids, and atherosclerotic plaque volume was carried out for all mice in all treatment groups using Pearson's correlation coefficients. Spots with densities that differed significantly either due to treatment $(P<0.05)$ or were highly correlated with $\mathrm{B}$ vitamin and lipid status at the end of the study $(P<0.001)$ were excised from the gel and identified by LC MS/MS. Association networks between biochemical biomarkers and protein expression were displayed using Cytoscape software (version 2.8.3; http://www.cytoscape.org). Principal component analysis (PCA) of the physiological biomarkers measured from each mouse in the study was carried out and the component scores calculated.

\section{Results}

B vitamin, homocysteine, lipid status and aorta plaque volume

Principal component analysis (PCA) of biochemical data for all animals revealed that the treatment phenotypes
Fig. 1 Primary component analysis (PCA) score plot of blood and tissue biomarkers significantly altered by folic acid, B vitamins and fat intake in ApoE null mice. Mice were fed a control $(C)$ or high-fat $(H F)$ diet depleted of folic acid $(\mathrm{F}-)$ or folic acid and vitamins $\mathrm{B}_{6}$ and $\mathrm{B}_{12}(\mathrm{~F}-\mathrm{B}-)$ for 16 weeks. Symbols represent individual replicate animals within every treatment group

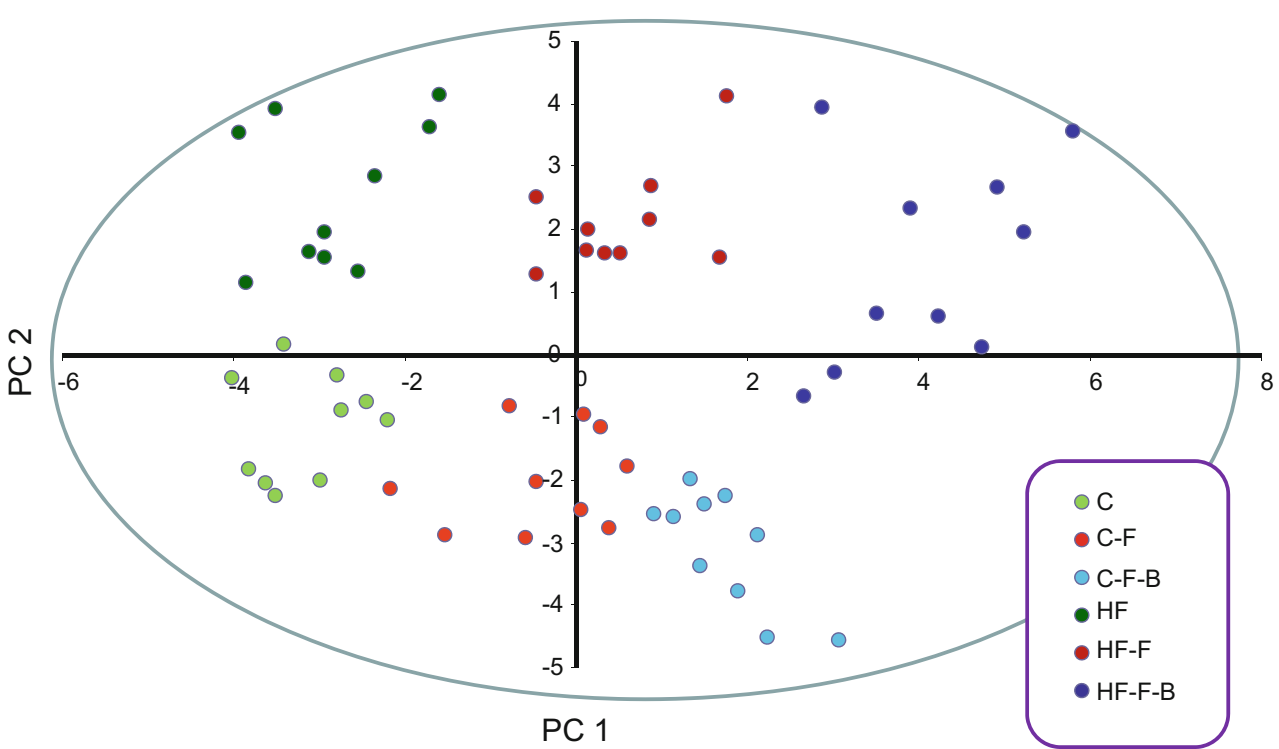


Table 1 The effect of folic acid, B vitamins and dietary fat intake on B vitamin, homocysteine and plasma and aorta adventitial lipid status in ApoE null mice

\begin{tabular}{|c|c|c|c|c|c|c|}
\hline \multirow[t]{2}{*}{ Biomarkers } & \multicolumn{3}{|l|}{ Control fat } & \multicolumn{3}{|l|}{ High fat } \\
\hline & $\mathrm{C}$ & $\mathrm{C}-\mathrm{F}$ & $\mathrm{C}-\mathrm{F}-\mathrm{B}$ & $\mathrm{HF}$ & $\mathrm{HF}-\mathrm{F}$ & $\mathrm{HF}-\mathrm{F}-\mathrm{B}$ \\
\hline \multicolumn{7}{|l|}{$B$ vitamin status } \\
\hline Plasma folate (ng/ml) & $87.8 \pm 4.8^{\mathrm{a}}$ & $16.2 \pm 1.5^{\mathrm{b}}$ & $14.9 \pm 0.9^{\mathrm{b}}$ & $104.9 \pm 5.5^{\mathrm{c}}$ & $15.5 \pm 1.7^{\mathrm{b}}$ & $15.0 \pm 1.4^{\mathrm{b}}$ \\
\hline Whole blood folate $(\mathrm{ng} / \mathrm{ml})$ & $498.9 \pm 42.9^{\mathrm{a}}$ & $163.5 \pm 8.9^{\mathrm{b}}$ & $184.7 \pm 6.8^{\mathrm{b}}$ & $507.9 \pm 26.0^{\mathrm{a}}$ & $149.6 \pm 9.7^{\mathrm{b}}$ & $193.3 \pm 18.7^{\mathrm{b}}$ \\
\hline Plasma $B_{12}(\mathrm{ng} / \mathrm{ml})$ & $24.8 \pm 2.3^{\mathrm{a}}$ & $15.3 \pm 1.0^{\mathrm{b}}$ & $6.8 \pm 0.1^{\mathrm{c}}$ & $25.6 \pm 3.0^{\mathrm{a}}$ & $12.5 \pm 0.5^{\mathrm{d}}$ & $6.1 \pm 0.5^{\mathrm{c}}$ \\
\hline Whole blood $\mathrm{B}_{12}(\mathrm{ng} / \mathrm{ml})$ & $22.5 \pm 1.0^{\mathrm{a}, \mathrm{c}}$ & $22.0 \pm 0.6^{\mathrm{a}, \mathrm{c}}$ & $7.2 \pm 0.7^{\mathrm{b}}$ & $24.9 \pm 1.6^{\mathrm{a}}$ & $20.5 \pm 1.1^{\mathrm{c}}$ & $6.9 \pm 1.0^{\mathrm{b}}$ \\
\hline Plasma homocysteine $(\mu \mathrm{M})$ & $7.8 \pm 0.4^{\mathrm{a}}$ & $18.1 \pm 1.9^{\mathrm{b}}$ & $28.4 \pm 3.6^{\mathrm{c}}$ & $6.7 \pm 0.3^{\mathrm{a}}$ & $14.2 \pm 1.0^{\mathrm{b}}$ & $45.5 \pm 4.3^{\mathrm{d}}$ \\
\hline \multicolumn{7}{|l|}{ Plasma lipids } \\
\hline Total cholesterol $(\mathrm{mmol} / \mathrm{L})$ & $12.7 \pm 0.51^{\mathrm{a}}$ & $10.9 \pm 0.61^{\mathrm{b}}$ & $12.9 \pm 0.40^{\mathrm{a}, \mathrm{b}}$ & $26.2 \pm 1.92^{\mathrm{c}}$ & $26.5 \pm 0.96^{\mathrm{c}}$ & $24.9 \pm 1.34^{\mathrm{c}}$ \\
\hline HDL cholesterol (mmol/L) & $5.22 \pm 0.33^{\mathrm{a}}$ & $5.51 \pm 0.84^{\mathrm{a}}$ & $5.71 \pm 0.92^{\mathrm{a}}$ & $9.23 \pm 0.40^{\mathrm{b}}$ & $10.42 \pm 0.87^{\mathrm{b}}$ & $8.86 \pm 0.59^{\mathrm{b}}$ \\
\hline LDL cholesterol (mmol/L) & $9.25 \pm 0.38^{\mathrm{a}}$ & $8.04 \pm 0.42^{\mathrm{b}}$ & $8.90 \pm 0.52^{\mathrm{a}, \mathrm{b}}$ & $24.19 \pm 1.85^{\mathrm{c}}$ & $24.79 \pm 1.11^{\mathrm{c}}$ & $22.58 \pm 1.06^{\mathrm{c}}$ \\
\hline Triglycerides (TG) (mmol/L) & $1.15 \pm 0.05^{\mathrm{a}, \mathrm{c}}$ & $1.27 \pm 0.08^{\mathrm{a}}$ & $2.20 \pm 0.25^{\mathrm{b}}$ & $0.89 \pm 0.14^{\mathrm{c}, \mathrm{e}}$ & $1.01 \pm 0.08^{\mathrm{c}, \mathrm{d}, \mathrm{e}}$ & $0.91 \pm 0.07^{\mathrm{e}}$ \\
\hline \multicolumn{7}{|l|}{ Aorta adventitial lipids } \\
\hline $\begin{array}{l}\text { Total cholesterol } \\
\text { (mmol/g lipid) }\end{array}$ & $35.1 \pm 4.7^{\mathrm{a}, \mathrm{c}}$ & $24.1 \pm 2.1^{\mathrm{b}}$ & $33.0 \pm 3.0^{\mathrm{a}}$ & $43.1 \pm 4.6^{\mathrm{a}, \mathrm{c}}$ & $53.4 \pm 8.4^{\mathrm{c}, \mathrm{d}}$ & $73.4 \pm 9.1^{\mathrm{d}}$ \\
\hline $\begin{array}{l}\text { HDL cholesterol } \\
\text { (mmol/g lipid) }\end{array}$ & ND & ND & ND & $1.0 \pm 1.0$ & $1.6 \pm 1.4$ & $6.1 \pm 4.1$ \\
\hline NEFA (mmol/g lipid) & $6.9 \pm 0.9^{\mathrm{a}}$ & $5.8 \pm 0.8^{\mathrm{a}}$ & $7.5 \pm 1.3^{\mathrm{a}, \mathrm{b}}$ & $7.5 \pm 0.8^{\mathrm{a}, \mathrm{b}}$ & $9.1 \pm 1.4^{\mathrm{a}, \mathrm{b}}$ & $10.2 \pm 1.0^{\mathrm{b}}$ \\
\hline $\begin{array}{l}\text { Triglycerides (TG) } \\
(\mu \mathrm{mol} / \mathrm{g} \text { lipid })\end{array}$ & $919.0 \pm 35.0^{\mathrm{a}, \mathrm{b}}$ & $849.0 \pm 58.7^{\mathrm{a}, \mathrm{c}}$ & $880.6 \pm 45.3^{\mathrm{a}, \mathrm{b}, \mathrm{c}}$ & $856.9 \pm 61.6^{\mathrm{a}, \mathrm{b}, \mathrm{c}}$ & $1,032.7 \pm 63.1^{\mathrm{b}}$ & $798.7 \pm 23.1^{\mathrm{c}}$ \\
\hline SATFAs (\% total fat) & $31.14 \pm 1.15^{\mathrm{a}, \mathrm{b}}$ & $30.84 \pm 0.69^{\mathrm{a}}$ & $33.38 \pm 0.84^{\mathrm{b}}$ & $27.84 \pm 0.59^{\mathrm{c}}$ & $28.81 \pm 0.46^{\mathrm{c}}$ & $33.89 \pm 0.49^{\mathrm{b}, \mathrm{d}}$ \\
\hline MUFAs (\% total fat) & $47.38 \pm 0.08^{\mathrm{a}, \mathrm{b}}$ & $47.36 \pm 0.44^{\mathrm{a}, \mathrm{b}}$ & $46.11 \pm 0.49^{\mathrm{a}}$ & $48.33 \pm 0.67^{\mathrm{b}}$ & $48.60 \pm 0.55^{\mathrm{b}, \mathrm{c}}$ & $42.45 \pm 0.69^{\mathrm{d}}$ \\
\hline PUFAs ( $\%$ total fat) & $20.76 \pm 1.02^{\mathrm{a}, \mathrm{b}}$ & $21.10 \pm 0.70^{\mathrm{a}, \mathrm{b}}$ & $19.56 \pm 0.84^{\mathrm{a}}$ & $22.53 \pm 0.54^{\mathrm{b}}$ & $21.77 \pm 0.30^{\mathrm{b}, \mathrm{c}}$ & $22.61 \pm 0.64^{\mathrm{b}, \mathrm{d}}$ \\
\hline $\begin{array}{l}\text { Aorta plaque volume } \\
(\mathrm{nM} / \mathrm{mg} \text { tissue })\end{array}$ & $0.274 \pm 0.014^{\mathrm{a}}$ & $0.260 \pm 0.018^{\mathrm{a}}$ & $0.261 \pm 0.007^{\mathrm{a}}$ & $0.556 \pm 0.024^{\mathrm{b}}$ & $0.637 \pm 0.027^{\mathrm{c}}$ & $0.602 \pm 0.030^{\mathrm{b}, \mathrm{c}}$ \\
\hline
\end{tabular}

These data are reproduced with the kind permission of the journals, Clinical Epigenetics (McNeil et al. 2011) and Molecular Nutrition and Food Research (McNeil et al. 2012)

Mice were fed a control (C) or high-fat (HF) diet depleted of folic acid (F-) or folic acid and vitamins $\mathrm{B}_{6}$ and $\mathrm{B}_{12}$ (F-B-) for 16 weeks. Values are mean \pm SEM for $n=9-10$ mice sampled per group. Biochemical data were analysed by two-way ANOVA for the effect of fat intake, vitamin composition and interaction between these factors. Significant differences between all groups were detected using the least significant difference (LSD) post hoc method. Data not sharing superscript letters differ by $P<0.05$. $B_{12}$ vitamin $\mathrm{B}_{12}, H D L$ high-density lipoprotein, $L D L$ low-density lipoprotein, MUFAs monounsaturated fatty acids, PUFAs polyunsaturated fatty acids, NEFA non-esterified fatty acids, SATFAs saturated fatty acids

separated differentially according to fat intake and folate and $\mathrm{B}$ vitamin treatment (Fig. 1). Aortic plaque volume, blood and lipid biomarkers are shown in Table 1. Folate was depleted more than $65 \%$ in mice fed a folate-deficient diet. $\mathrm{B}_{12}$ decreased more than $70 \%$ in animals fed the combined folate and B vitamin-depleted diet. Folate deficiency increased homocysteine twofold. Homocysteine was elevated further in mice fed a combined B vitamindepleted diet (approx. fourfold). $\mathrm{B}_{12}$ depletion and hyperhomocysteinemia were exacerbated by high fat. Total cholesterol was increased in aorta adventitial lipid in response to a high-fat diet. Plasma cholesterol, HDL and LDL were elevated (approx. twofold). Folate depletion alone did not impact on circulating or adventitial lipids. However, feeding a combined folate and B vitamin-deficient diet exacerbated cholesterol accumulation and significantly reduced MUFA and increased pro-atherogenic SATFAs in the aorta adventitial lipid. Plaque volume was increased almost twofold $(P<0.0001)$ in mice fed a $\mathrm{HF}$ diet for 16 weeks when compared with animals on a control diet (Table 1). Plaque volume was significantly increased $(17 \% ; P<0.05)$ in mice depleted of folic acid. Imposing a combined B vitamin deficiency did not increase plaque development further (Table 1).

Atherosclerotic plaque abundance was correlated strongly with circulating and vascular lipid levels, and weakly with plasma vitamin $\mathrm{B}_{12}$ (Table 2). Plaque formation did not correlate directly either with blood folate or homocysteine. However, whole blood and plasma $\mathrm{B}_{12}$ did strongly influence both circulating and adventitial lipid concentrations, with low $\mathrm{B}_{12}$ associated with high levels of plasma TG, adventitial total cholesterol and SATFAs and, 
conversely, a lower proportion of MUFAs. Low folate was similarly associated with accumulation of SATFAs in the aorta adventitia. Hyperhomocysteinemia was positively associated with elevated SATFA and reduced MUFA concentrations in the adventitia (Table 2).

\section{Aorta tissue proteomics}

Comparative quantitative proteomics revealed approximately 800 spots in the aorta samples that matched across all 60 treatment gels. The primary proteins (approx. $35 \%$ of total identified proteins) influenced by treatment in the aorta were involved in regulating cytoskeletal organisation, cell adhesion and cell motility (Fig. 2). Mean (and group SEM) spot densities for aorta proteins that differed between treatment groups $(P<0.05)$ are shown in Table 3 . The independent effects of fat or vitamin treatment on protein expression are described ( $P$ values) together with the synergistic effect of a $\mathrm{HF}$ and combined vitamindepleted diet. The effect of $\mathrm{B}_{6}$ and $\mathrm{B}_{12}$ depletion, above folate deficiency alone is also shown. Vitamin depletion alone altered expression of only six aorta proteins [fibrinogen, gelsolin, glutamate dehydrogenase 1, lactate dehydrogenase and talin-1 (all upregulated) and peptidyl-prolyl cis-trans isomerise A (downregulated)]. Feeding a combined vitamin-deficient and high-fat diet had a strong synergistic effect on 25 aorta proteins (including dermatopontin, fibrinogen, glutathione $S$-transferase $\mathrm{Mu} 1$, peroxiredoxin-2, serotransferrin, transgelin and vimentin; Table 3).

Correlation analysis revealed strong $(P<0.001$; mean $q=0.03$ ) associations between lipid and vitamin status, and aorta protein expression (Table 4). Protein expression in the aorta was exclusively correlated with aorta adventitial lipid levels (total fat, cholesterol, HDL, NEFA) and with the proportion of saturated and monounsaturated fat. Figure 3 constructed from biochemical and aorta protein correlations demonstrates the impact that B vitamins, homocysteine and methionine cycle intermediates have on lipoprotein accumulation directly in aorta adventitial lipid (e.g., increased SATFAs, decreased MUFAs, increased total cholesterol) and the influence that aorta lipid levels have on the expression of more than 80 aorta proteins (identified by spot number in Supplementary Table 1).

\section{Discussion}

The primary aim of this study was to examine and quantify the differential effects of folate and/or B vitamin depletion on global protein expression in the aorta of ApoE mice and to consider the role of these proteins on cellular processes implicated in human vascular disease. Feeding a folate and/
Table 2 Correlation analyses of B vitamin, lipid status and atherosclerotic plaque formation in ApoE null mice

\begin{tabular}{|c|c|c|c|}
\hline \multicolumn{2}{|l|}{ Biomarker } & Correlation & $P$ value \\
\hline \multicolumn{4}{|l|}{ Plaque associations } \\
\hline Plaque abundance & Plasma HDL & 0.763 & $8 \mathrm{E}-12$ \\
\hline Plaque abundance & Plasma LDL & 0.736 & $5 \mathrm{E}-10$ \\
\hline Plaque abundance & $\begin{array}{r}\text { Plasma total } \\
\text { cholesterol }\end{array}$ & 0.708 & $\begin{array}{c}1.3 \mathrm{E}- \\
08\end{array}$ \\
\hline Plaque abundance & $\begin{array}{l}\text { Adventitia total } \\
\text { cholesterol }\end{array}$ & 0.586 & $\begin{array}{c}2.1 \mathrm{E}- \\
06\end{array}$ \\
\hline Plaque abundance & Adventitia HDL & 0.446 & 0.00055 \\
\hline Plaque abundance & Plasma TG & -0.427 & 0.00106 \\
\hline Plaque abundance & Adventitia SATFA & 0.374 & 0.00526 \\
\hline Plaque abundance & Plasma $B_{12}$ & -0.271 & 0.04303 \\
\hline \multicolumn{4}{|c|}{$B$ vitamin status and lipid metabolism } \\
\hline $\begin{array}{l}\text { Adventitia total } \\
\text { cholesterol }\end{array}$ & Plasma $B_{12}$ & -0.339 & 0.00802 \\
\hline Adventitia SATFA & Whole blood $\mathrm{B}_{12}$ & -0.632 & $\begin{array}{c}1.3 \mathrm{E}- \\
07\end{array}$ \\
\hline Adventitia SATFA & $\begin{array}{l}\text { Plasma } \\
\text { homocysteine }\end{array}$ & 0.534 & $\begin{array}{c}1.9 \mathrm{E}- \\
05\end{array}$ \\
\hline Adventitia SATFA & Plasma $B_{12}$ & -0.502 & $\begin{array}{l}6.8 \mathrm{E}- \\
05\end{array}$ \\
\hline Adventitia SATFA & Plasma folate & -0.396 & 0.00231 \\
\hline Adventitia MUFA & $\begin{array}{l}\text { Plasma } \\
\text { homocysteine }\end{array}$ & -0.564 & $\begin{array}{c}4.8 \mathrm{E}- \\
06\end{array}$ \\
\hline Adventitia MUFA & Plasma $B_{12}$ & 0.456 & 0.00037 \\
\hline Adventitia MUFA & Whole blood $\mathrm{B}_{12}$ & 0.445 & 0.00052 \\
\hline
\end{tabular}

Analysis of associations between blood B vitamin status markers and blood, vascular lipids and plaque formation were carried out for all mice in all treatment groups using Pearson's correlation coefficients. Biomarker and strength of associations (Pearson's correlation coefficient; positive and negative) are shown together with the corresponding $P$ value. $B_{12}$ vitamin $\mathrm{B}_{12}, H D L$ high-density lipoprotein, $L D L$ low-density lipoprotein, MUFAs monounsaturated fatty acids, PUFAs polyunsaturated fatty acids, NEFA non-esterified fatty acids, SATFAs saturated fatty acids

or B vitamin depleted diet against a control fat background altered expression of only very few aorta protein [fibrinogen, gelsolin, lactate dehydrogenase, talin (upregulated) and peptidyl-prolyl cis-trans isomerise (decreased)]. However, when combined with a major dietary challenge (a high-fat diet), B vitamin depletion strongly perturbed many more processes [including oxidative stress, inflammation and vascular smooth muscle cell (VSMC) activation and migration] causally associated both with rodent and human atherosclerosis (see Fig. 2). Suboptimum folate status and hyperhomocysteinemia are linked with hyperlipidemia and oxidative stress, major risk factors for vascular disease (Kris-Etherton et al. 2002; Lohm et al. 2002; Mikael et al. 2006; Devlin et al. 2007). Here, B vitamin depletion was associated with significant changes in vascular adventitial lipid metabolism and protein markers of 


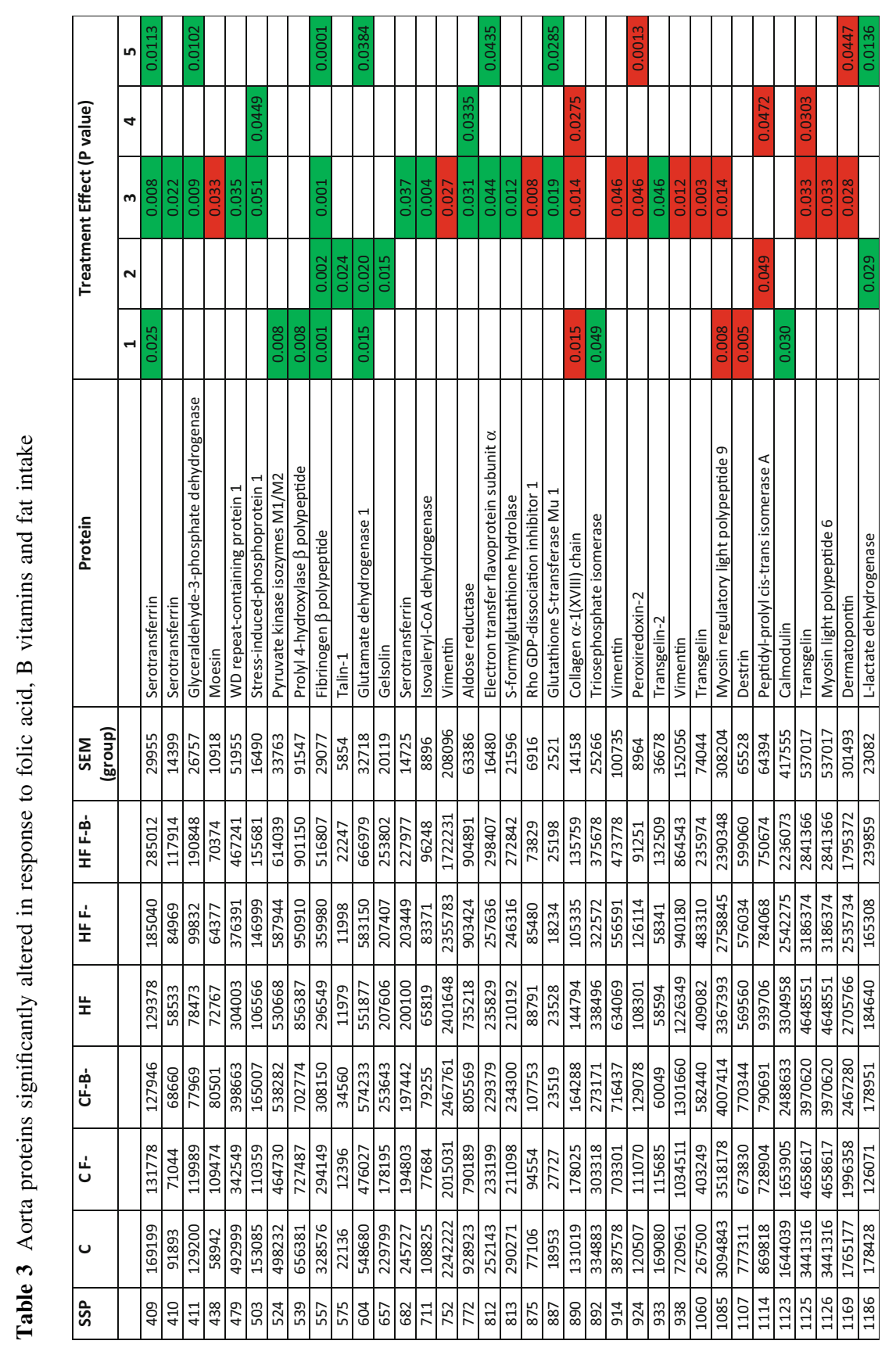

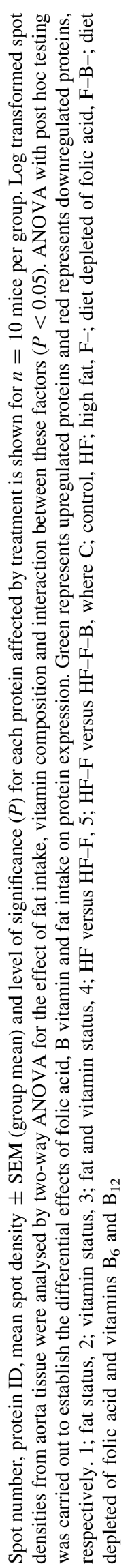


Table 4 Associations (correlation analyses) between aorta lipid and aorta protein expression
Analysis of associations between aorta tissue protein expression and vascular lipids was carried out for all mice in all treatment groups using Pearson's correlation coefficients. Lipid biomarker, spot number, protein name and strength of association (positive and negative) are shown together with the corresponding $P$ value. $B_{12}$ vitamin $\mathrm{B}_{12}, H D L$ high-density lipoprotein, $L D L$ low-density lipoprotein, MUFAs monounsaturated fatty acids, PUFAs polyunsaturated fatty acids, NEFA non-esterified fatty acids, SATFAs saturated fatty acids

\begin{tabular}{|c|c|c|c|c|}
\hline Biomarker & Spot & Protein & Correlation & $P$ value \\
\hline $\begin{array}{l}\text { Adventitia total } \\
\text { cholesterol }\end{array}$ & 829 & Annexin A1 & -0.500 & $4.69 \mathrm{E}-05$ \\
\hline $\begin{array}{l}\text { Adventitia total } \\
\text { cholesterol }\end{array}$ & 582 & $\begin{array}{l}\text { Protein-glutamine gamma- } \\
\text { glutamyltransferase } 2\end{array}$ & 0.413 & 0.00102 \\
\hline $\begin{array}{l}\text { Adventitia total } \\
\text { cholesterol }\end{array}$ & 912 & Isocitrate dehydrogenase & 0.418 & 0.00088 \\
\hline $\begin{array}{l}\text { Adventitia total } \\
\text { cholesterol }\end{array}$ & 524 & Pyruvate kinase isozymes M1/M2 & 0.421 & 0.00079 \\
\hline $\begin{array}{l}\text { Adventitia total } \\
\text { cholesterol }\end{array}$ & 468 & $78 \mathrm{kDa}$ glucose-regulated protein & 0.424 & 0.00072 \\
\hline $\begin{array}{l}\text { Adventitia total } \\
\text { cholesterol }\end{array}$ & 473 & Heat shock protein $1 \mathrm{~B}$ & 0.450 & 0.00030 \\
\hline $\begin{array}{l}\text { Adventitia total } \\
\text { cholesterol }\end{array}$ & 604 & Glutamate dehydrogenase 1 & 0.472 & 0.00013 \\
\hline $\begin{array}{l}\text { Adventitia total } \\
\text { cholesterol }\end{array}$ & 557 & Fibrinogen $\mathrm{b}$ polypeptide & 0.484 & $9.09 \mathrm{E}-05$ \\
\hline $\begin{array}{l}\text { Adventitia total } \\
\text { cholesterol }\end{array}$ & 812 & Electron transfer flavoprotein subunit $\alpha$ & 0.495 & $5.85 \mathrm{E}-05$ \\
\hline $\begin{array}{l}\text { Adventitia total } \\
\text { cholesterol }\end{array}$ & 409 & Serotransferrin & 0.545 & $6.68 \mathrm{E}-06$ \\
\hline Adventitia HDL & 557 & Fibrinogen b polypeptide & 0.461 & 0.00020 \\
\hline Adventitia HDL & 468 & $78 \mathrm{kDa}$ glucose-regulated protein & 0.556 & $4.02-06$ \\
\hline Adventitia HDL & 409 & Serotransferrin & 0.676 & $3.14 \mathrm{E}-09$ \\
\hline Adventitia HDL & 473 & Heat shock protein 1B & 0.687 & $1.30 \mathrm{E}-09$ \\
\hline Adventitia NEFA & 914 & Vimentin & -0.451 & 0.00030 \\
\hline Adventitia NEFA & 772 & Aldose reductase & 0.414 & 0.00101 \\
\hline Adventitia NEFA & 812 & Electron transfer flavoprotein subunit $\alpha$ & 0.443 & 0.00038 \\
\hline Adventitia NEFA & 468 & $78 \mathrm{kDa}$ glucose-regulated protein & 0.471 & 0.00014 \\
\hline Adventitia NEFA & 524 & Pyruvate kinase isozymes M1/M2 & 0.472 & 0.00014 \\
\hline Adventitia NEFA & 604 & Glutamate dehydrogenase 1 & 0.487 & $8.32 \mathrm{E}-05$ \\
\hline Adventitia NEFA & 912 & Isocitrate dehydrogenase & 0.497 & $5.45 \mathrm{E}-05$ \\
\hline Adventitia NEFA & 909 & GTP-binding nuclear protein Ran & 0.573 & $1.71 \mathrm{E}-06$ \\
\hline Adventitia MUFA & 557 & Fibrinogen $\beta$ polypeptide & -0.467 & 0.00024 \\
\hline Adventitia MUFA & 410 & Serotransferrin & -0.457 & 0.00035 \\
\hline Adventitia MUFA & 503 & Stress-induced-phosphoprotein 1 & -0.429 & 0.00085 \\
\hline Adventitia SATFA & 1,125 & Transgelin & -0.512 & $4.72 \mathrm{E}-05$ \\
\hline
\end{tabular}

glucose-related proteins and Hsp 1B) and stress-induced phosphoprotein 1 (a modulator of Hsp70 activity) were positively associated with total and HDL adventitial cholesterol and negatively linked with MUFA concentration. Rho GDP dissociation inhibitor 1 negatively regulates VSMC adhesion and enhances migration (Qi et al. 2008). Here, feeding a high-fat combined B vitamin-depleted diet significantly downregulated Rho GDP dissociation inhibitor 1 expression in mouse aorta. Conversely, GTP-binding nuclear protein Ran expression is positively associated with VSMC migration (Fuastino et al. 2010). GTP-binding nuclear protein Ran expression was upregulated in this experiment. Actin assembly and disassembly drives vascular cytoskeletal organisation and regulates VSMC phenotype. Many aorta proteins (including transgelin, vimentin, dermatopontin, collagen, moesin, myosin regulatory light polypeptide 6 and 9, and talin) that influence actin filament regulation, cell differentiation, cell adhesion and cell migration were altered by treatment in this study. Reduced expression of transgelin-1 $(\operatorname{sm} 22 \alpha)$ is related to VSMC proliferation and atherosclerotic plaque formation (Feil et al. 2004; Shen et al. 2010). Transgelin expression was strongly downregulated in the aorta of mice fed a highfat and combined B vitamin-depleted diet. Moreover, a strong negative relationship between transgelin and saturated fatty acid accumulation in adventitial lipid was observed. Overexpression of gelsolin increases vascular endothelial cell motility (Silacci et al. 2003). Gelsolin was 


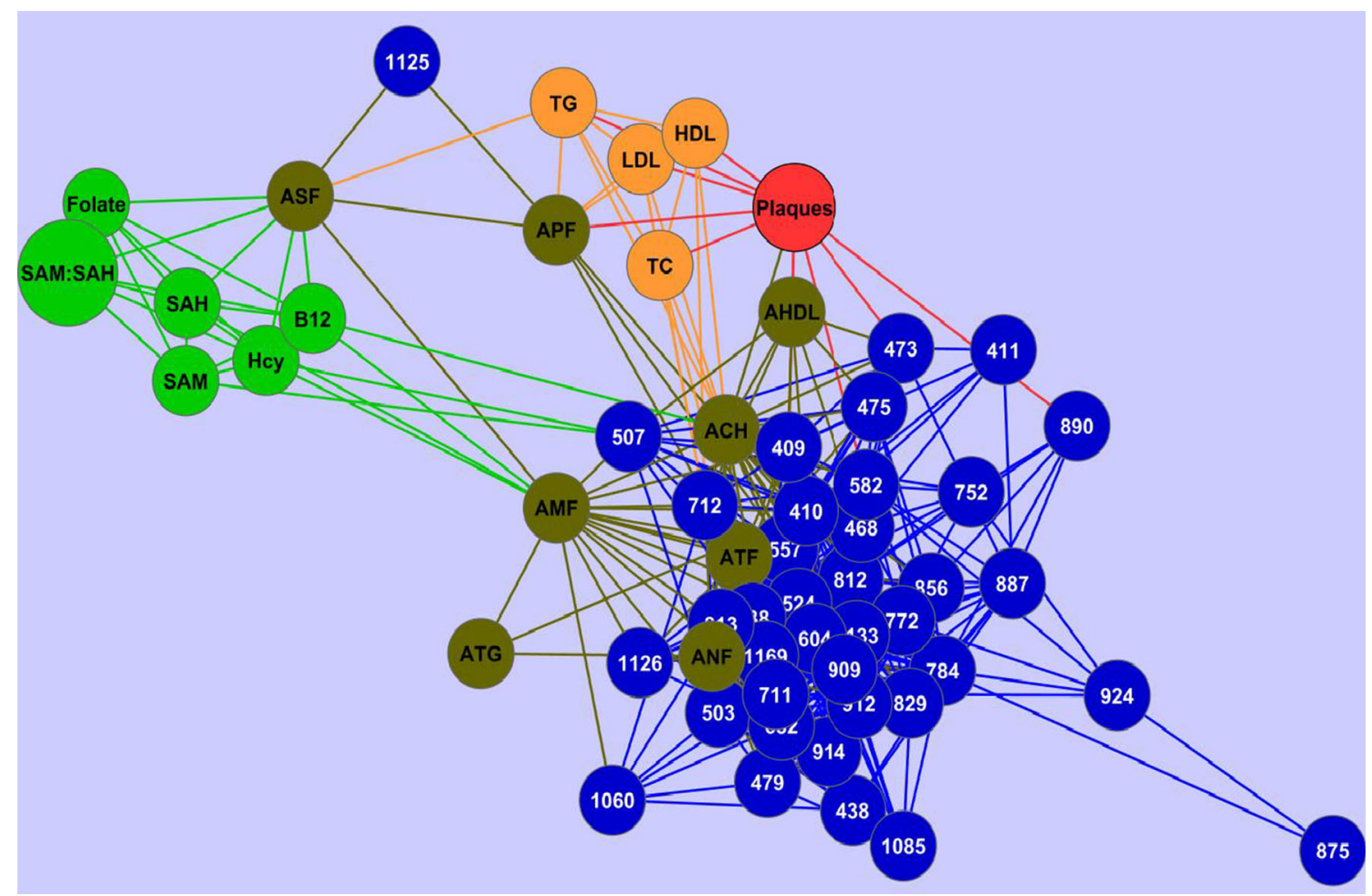

Fig. 3 Association network 1 (ball and stick) of aorta proteins influenced by B vitamin and fat intake in ApoE null mice. Association networks between biochemical biomarkers and protein expression were displayed using Cytoscape software (version 2.8.3; http://www. cytoscape.org). Lines connecting proteins and biomarkers indicate a correlation with $R>0.3$ or $<-0.3$. ACH adventitial total cholesterol, $A H D L$ adventitial high-density lipoprotein, $A M F$ adventitial monounsaturated fatty acid, $A N F$ adventitial non-esterified fatty acid, $A P F$ adventitial polyunsaturated fatty acid, $A S F$ adventitial saturated fatty acid, $A T F$ adventitial total fat, $A T G$ adventitial triglyceride, $B_{12}$ vitamin $\mathrm{B}_{12}, H D L$ high-density lipoprotein, $H c y$ homocysteine, $L D L$ low-density lipoprotein, $S A H S$-adenosylhomocysteine, $S A M S$ adenosylmethionine, SAM: $S A H S$-adenosylmethionine: $S$-adenosylhomocysteine ratio, $T C$ total cholesterol, $T G$ triglyceride. Spot number and protein name: 409, serotransferrin; 410, serotransferrin; 411,

elevated in the aorta of ApoE null mice in this study. While collagen deposition is associated both with plaque formation and stability, polymerised collagen type 1 maintains VSMC quiescence (Adiguzel et al. 2009). Here, expression of this anti-proliferative and anti-inflammatory protein was significantly decreased. Dermatopontin, an extracellular matrix protein, regulates collagen deposition and promotes cell adhesion (Okamoto et al. 2010), while moesin, a member of the ezrin/radixin/moesin (ERM) protein family (together with Rho GDP dissociation inhibitor 1), inhibits cell migration (Takahashi et al. 1997). Feeding a high-fat B vitamin-depleted diet decreased aorta moesin and dermatopontin levels and (as described previously) Rho GDP dissociation inhibitor 1 expression. Conversely, expression of talin, a cytoskeletal protein which promotes cell dispersal (Albiges-Rizo et al. 1995) was doubled. glyceraldehyde-3-phosphate dehydrogenase; 438, moesin; 468, $78 \mathrm{kDa}$ glucose-regulated protein; 473 , heat shock protein $1 \beta, 479$, WD repeat-containing protein $1 ; 503$, stress-induced-phosphoprotein $1 ; 507$, transforming growth factor $\beta$-induced; 524 , pyruvate kinase isozymes M1/M2; 557, fibrinogen B $\beta$ polypeptide isoforms; 582, protein-glutamine gamma-glutamyltransferase 2; 604, glutamate dehydrogenase 1; 682, serotransferrin; 711, isovaleryl-CoA dehydrogenase; 752, vimentin; 772, aldose reductase; 812, electron transfer flavoprotein subunit $\alpha ; 813, S$-formylglutathione hydrolase; 829, annexin A1; 875, Rho GDP dissociation inhibitor 1; 887, glutathione $S$-transferase $\mathrm{Mu} 1$; 890, collagen $\alpha$-1(XVIII) chain; 909, GTP-binding nuclear protein Ran; 912, isocitrate dehydrogenase; 924, peroxiredoxin-2; 938, vimentin; 1,060, transgelin; 1,085, myosin regulatory light polypeptide $9 ; 1,125$, transgelin; 1,126 , myosin light polypeptide $6 ; 1,169$, dermatopontin

These parallel quantitative changes in numerous proteins implicated in VSMC proliferation and motility provide strong but indirect evidence that feeding a combined $\mathrm{B}$ vitamin-depleted high-fat diet may promote an invasive VSMC phenotype in mice vivo. Whether B vitamin depletion impacts on human aorta protein expression and on SMC phenotype in the same way remains to be established. ApoE null mice are recognised as a good model for human vascular disease, spontaneously developing atherosclerotic plaques morphologically similar to lesions found in humans (Zhang et al. 2002). Additionally, here, we have employed a nutritionally relevant nutritional rodent model that better reflects a moderate B vitamin deficiency when compared with previous studies inducing supraphysiologically high levels of homocysteine (McNeil et al. 2011). Furthermore, our findings are consistent with 
other studies using proteomic technologies to profile aorta or plasma protein expression patterns in several human vascular pathologies. Many proteins, acknowledged as characteristic of vascular disease progression, are identified in this animal study as strongly linked to nutritional B vitamin depletion (noted in parentheses). Medial degeneration in the human ascending aorta alters the expression of proteins critical in vascular remodelling, cytoskeleton organisation and muscle contraction (actin, collagen, profilin, myosin, vimentin), VSMC phenotype (transgelins), protein folding and ROS detoxification (Hsps, peroxiredoxin, protein disulphide isomerase), and iron transport and inflammation (serotransferrin) (Mayr et al. 2005). The HUPO Plasma Proteome Project identified more than 300 human plasma proteins as strong biomarkers of vascular function and/or disease. These include markers of inflammation (transferrin), coagulation (fibrinogen A $\alpha$-polypetide, fibronectin) and cytoskeletal organisation (actin, myosin, talin and villin) (Sanzen et al. 2010). Protein profiling has also been employed to investigate mechanism of vascular dysfunction in animal models. Serotransferrin, $78 \mathrm{kDa}$ glucose-regulated protein and pyruvate kinase isozyme M1/M2 are elevated in experimentally induced rat heart failure (Ingwall and Weiss 2004), while fibrinogen, collagen, peroxidase and transgelin are overexpressed in atherosclerotic mice (Mayr et al. 2005; Wu et al. 2007). Transgelin-1 is increased in the artery wall of hypertensive rats (Delbosc et al. 2008).

In summary, nutritional B vitamin depletion when combined with a high-fat diet in a mouse model of human vascular disease quantifiably altered the expression of many aorta proteins implicated in human atherosclerosis, primarily proteins associated with regulating the VSMC phenotype. It remains to be established whether these significant changes in vascular protein expression are driven by elevated homocysteine or are a consequence of other B vitamin-sensitive mechanisms.

Acknowledgments This work was funded by The Scottish Government Rural and Environment Science and Analytical Services Division (RESAS).

Conflict of interest All institutional and national guidelines for the care and use of laboratory animals were followed. Susan Duthie, John H Beattie, Margaret-J Gordon, Lynn P Pirie, Fergus Nicol, Martin D Reid, Gary J Duncan, Louise Cantlay, Graham Horgan and Christopher J McNeil declare that they have no conflict of interest.

\section{References}

Adiguzel E, Ahmad PJ, Franco C, Bendeck MP (2009) Collagens in the progression and complications of atherosclerosis. Vasc Med $14: 73-89$
Albiges-Rizo C, Frachet P, Block MR (1995) Down regulation of talin alters cell adhesion and the processing of the $\alpha 5 \beta 1$ integrin. J Cell Sci 108:3317-3329

Almofti MR, Huang Z, Yang P, Rui Y (2006) Proteomic analysis of rat aorta during atherosclerosis induced injection by high cholesterol diet and injection of vitamin D3. Clin Exp Pharmacol Physiol 33:305-309

Beattie JH, Duthie SJ, Kwunm I-S, Ha T-Y (2009) Rapid quantification of aortic lesions in ApoE-/-mice. J Vasc Res 46:347-352

Brownlee M (2001) Biochemistry and molecular cell biology of diabetic complications. Nature 414:813-820

Burdge GC, Wright P, Jones AE, Wooten SA (2000) A method for separation of phosphatidylcholine, triacylglycerol, non-esterified fatty acids and cholesterol esters from plasma by solid phase extraction. B J Nutr 84:781-787

Cheuk BL, Cheng SW (2011) Annexin A1 expression in atherosclerotic carotid plaques and its relationship with plaque characteristics. Eur J Vasc Endovasc Surg 41:364-371

Delbosc S, Haloui M, Louedec L, Dupuis M et al (2008) Proteomic analysis permits the identification of new biomarkers of arterial wall remodelling in hypertension. Mol Med 14:383-394

Devlin AM, Singh R, Wade RE, Innis SM et al (2007) Hypermethylation of Fads2 and altered hepatic fatty acid and phospholipid metabolism in mice with hyperhomocysteinemia. J Biol Chem 282:37082-37090

Durga J, Bots ML, Schouten EG, Kok FJ (2005) Low concentrations of folate not hyperhomocysteinemia are associated with carotid intima-media thickness. Atherosclerosis 179:285-292

Duthie SJ, Mavrommatis Y, Rucklidge G, Reid M et al (2008) The response of human colonocytes to folate deficiency in vitro: functional and proteomic analysis. J Proteome Res 7:3254-3266

Farina A, Chambrey A, Esposito S, Agozzino L et al (2010) Proteomic profiling of medial degeneration in human ascending aorta. Clin Biochem 43:387-396

Feil S, Hofmann F, Feil R (2004) SM22 $\alpha$ modulates vascular smooth muscle cell phenotype during atherogenesis. Circ Res 94:863-865

Folch J, Less M, Sloane-Stanley GH (1957) A simple method for the isolation and purification of total lipids from animal tissues. J Biol Chem 226:497-509

Fuastino RS, Stronger LNW, Richard MN, Czubryt MP, Ford DA et al (2010) RanGAP-mediated nuclear protein import in vascular smooth muscle cells is augmented by lysophosphatidylcholine. Mol Pharmacol 71:438-445

Gleissner CA, Sanders JM, Nadler J, Ley K (2008) Upregulation of aldose reductase during foam cell formation as possible link between diabetes, hyperlipidemia and atherosclerosis. Arterioscler Thromb Vasc Biol 28:1137-1143

Ingwall JS, Weiss RG (2004) Is the failing heart energy starved? On using chemical energy to support cardiac function. Circ Res 95:135-145

Kofoed SC, Wittrup HH, Sillesen H, Nordestgaard BG (2003) Fibrinogen predicts ischemic stroke and advanced atherosclerosis but not echolucent, rupture-prone carotid plaques. The Copenhagen City Heart Study. Eur Heart J 24:567-576

Kris-Etherton PM, Etherton TD, Carlson J, Gardner C (2002) Recent discoveries in inclusive food-based approaches and dietary patterns for reduction in risk for cardiovascular disease. Curr Opin Lipidol 13:397-407

Lohm M, Dubrovska G, Lauterbach B, Luftm FC et al (2002) Perivascular fat releases a vascular relaxing factor. FASEB J 16:1057-1063

Mangoni AA, Sherwood RA, Asonganyi B, Swift CG (2005) Shortterm folic oral acid supplementation enhances endothelial 
function in patients with type 2 diabetes. Am J Hypertens $81: 220-226$

Mayr M, Chung Y-I, Mayr U, Yin X et al (2005) Proteomic and metabolic analyses of atherosclerotic vessels from apolipoprotein E-deficient mice reveal alterations in inflammation, oxidative stress and energy metabolism. Arterioscler Thromb Vasc Biol 25:2135-2142

McNeil CJ, Beattie JH, Gordon M-J, Pirie LP et al (2011) Differential effects of nutritional folic acid deficiency and moderate hyperhomocysteinemia on aortic plaque formation and genome-wide DNA methylation in vascular tissue from ApoE-/-mice. Clin Epigenet 2:361-368

McNeil CJ, Beattie JH, Gordon M-J, Pirie LP et al (2012) Nutritional B vitamin deficiency disrupts lipid metabolism causing accumulation of proatherogenic lipoproteins in the aorta adventitia of ApoE null mice. Mol Nutr Food Res 56:1122-1130

Mikael LG, Genest J, Rozen R (2006) Elevated homocysteine reduces apolipoprotein A-1 expression in hyperhomocysteinemic mice and in males with coronary artery disease. Circ Res 98:564-571

Okamoto O, Hozumi K, Katagiri F, Takahashi N et al (2010) Dermatopontin promotes epidermal keratinocyte adhesion via $\alpha 3 \beta 1$ integrin and a proteoglycan receptor. Biochemistry 49:147-155

Perretti M, Dalli J (2009) Exploiting the annexin A1 pathway for the development of novel anti-inflammatory therapeutics. Brit $\mathrm{J}$ Pharmacol 158:936-946

Pockley AG, Georgiades A, Thulin T, de Faire U et al (2003) Serum heat shock protein 70 levels predict the development of atherosclerosis in subjects with established hypertension. Hypertension 42:235-238

Qi Y-X, Qu M-J, Long D-K, Liu B et al (2008) Rho-GDP dissociation inhibitor alpha down regulated by low shear stress promotes vascular smooth muscle cell migration and apoptosis: a proteomic analysis. Cardiovasc Res 80:114-122

Sabeti S, Exner M, Mlekusch W, Amighi J et al (2005) Prognostic impact of fibrinogen in carotid atherosclerosis: non-specific indicator of inflammation or independent predictor of disease progression? Stroke 36:1400-1404

Sanzen Y, Ito M, Ohta M, Yoshida Y et al (2010) Functional proteomic analysis of experimental autoimmune myocarditisinduced chronic heart failure in the rat. Biol Pharm Bull 33:477-486

Shen J, Yang M, Ju D, Jiang H et al (2010) Disruption of SM22 promotes inflammation after artery injury via nuclear factor $\mathrm{k} \beta$ activation. Circ Res 106:1351-1362
Silacci P, Mazzolai L, Gauci C, Stergiopulos N et al (2003) Gelsolin superfamily proteins: key regulators of cellular functions. Cell Mol Life Sci B 61:2614-2623

Smith EB (1995) Fibrinogen, fibrin and the arterial wall. Eur Heart J 16:11-15

Splaver A, Lamas GA, Hennekens CH (2004) Homocysteine and cardiovascular disease: biological mechanisms, observational epidemiology and the need for randomised trials. Am Heart $\mathbf{J}$ 148:34-40

Takahashi K, Sasaki T, Mammoto A, Takaishi K et al (1997) Direct interaction of the Rho GDP dissociation inhibitor with ezrin/ radixin/moesin initiates the activation of the Rho small $G$ protein. J Biol Chem 272:23371-23375

Tawakol A, Migrino RQ, Aziz KS, Waitkowska W et al (2005) High dose folic acid acutely improves coronary vasodilator functions in patients with coronary artery disease. J Am Coll Cardiol 45:1580-1584

Till U, Rohl P, Jentsch A, Till H (2005) Decrease of carotid intimamedia thickness in patients at risk to cerebral ischemia after supplementation with folic acid, vitamins $\mathrm{B}_{6}$ and $\mathrm{B}_{12}$. Atherosclerosis 181:131-135

Vikramadithyan RK, Hu Y, Noh H-L, Liang C-P et al (2005) Human aldose reductase expression accelerates diabetic atherosclerosis in transgenic mice. J Clin Invest 115:2434-2443

Voutilainen S, Lakka TA, Porkkala-Sarataho E, Rissanen T et al (2000) Low serum folate concentrations are associated with an excess incidence of acute coronary events: the Kuopio Ischaemic Heart Disease Risk Factor Study. Eur J Clin Nutr 54:424-428

Voutilainen S, Virtanen JK, Rissanen TH, Alfthan G et al (2004) Serum folate and homocysteine and the incidence of acute coronary events: the Kuopio Ischaemic Heart Disease Risk Factor Study. Am J Clin Nutr 80:317-323

Wu J, Liu W, Sousa E, Qiu Y et al (2007) Proteomic identification of endothelial proteins isolated in situ from atherosclerotic aorta via systemic perfusion. J Proteome Res 6:4728-4736

$\mathrm{Xu}$ Q (2002) Role of heat shock proteins in atherosclerosis. Arterioscler Thromb Vasc Biol 22:1547-1559

Zhang SH, Reddick RL, Piedrahita JA, Maeda N (2002) Spontaneous hypercholesterolemia and arterial lesions in mice lacking apolipoprotein E. Science 258:468-471 